\title{
Real-time ultrasound assessment of sternal stability in adhesive-enhanced sternal closure
}

\author{
Doa El-Ansary ${ }^{1 *}$, Sulakshana Balachandran ${ }^{1}$, Alistair Royse ${ }^{2}$, Kathryn King-Shier ${ }^{3,4}$, Adam Bryant $^{1}$, Linda Denehy $^{1}$ and Paul Fedak $^{4}$ \\ ${ }^{1}$ Department of Physiotherapy, The University of Melbourne, Victoria, Australia \\ ${ }^{2}$ Department of Surgery, Melbourne Health, Victoria, Australia \\ ${ }^{3}$ Faculty of Nursing, University of Calgary, Alberta, Canada \\ ${ }^{4}$ Department of Cardiac Science, University of Calgary, Libin Cardiovascular Institute of Alberta, Calgary, Canada
}

\begin{abstract}
Purpose: Conventional sternal closure by wire cerclage is the current standard of care worldwide but can result in pathologic sternal displacement and persistent sternal pain. The aim of this case report was to quantify sternal separation and sternal micro-motion in two patients following conventional wire cerclage alone (patient 1) and adhesive-enhanced closure on a platform of wire cerclage (patient 2) respectively.

Description: Sternal separation and micro-motion was measured using real-time ultrasound. Pain and sternal stability were assessed using a Visual Analogue Scale (VAS) and the Sternal Instability Scale (SIS) respectively.

Evaluation: Patient 1 demonstrated sternal separation and micro-motion that was evident in the coronal and sagittal planes during upper limb movements and cough. Patient 2 demonstrated no sternal separation or micro-motion post-operatively with cortical bone bridging the sternal edges.

Conclusions: Real-time ultrasound is a non-invasive, valid and reliable measurement tool for quantification of sternal separation and micro-motion; monitoring of sternal healing and evaluation of sternal closure techniques. Future research investigating sternal closure in particular adhesive-enhanced closure is warranted in a larger population.
\end{abstract}

\section{Background}

Conventional sternal closure by wire cerclage is the current standard of care worldwide but can result in pathologic sternal displacement and persistent sternal pain, particularlyin patients who increasingly present with multiple co-morbidities and conditions that compromise bone and wound healing [1-4]. This has prompted ongoing developments aimed at attaining optimal sternal closure (e.g. titanium plates) and interventions to facilitate recovery following deep wound infections (e.g. Vacuum-assisted closure (VAC) therapy) [57]. Most sternal closure techniques aim to provide optimal closure in the coronal plane to reduce distraction of the sternal halves $[3,4,7,8]$. However, previous studies have demonstrated that activity of the upper limb and trunk result in multi-planar motion of the sternal edges [8]. Augmenting sternal closure with techniques such as bone adhesiveshas been demonstrated to accomplish osteointegration and rigid bone fixation within 24 hours of application [1-3]. Prior research has illustrated itsbenefits on functional recovery, respiratory capacity and incisional pain $[1,3]$. However, the degree of sternal separation and micro-motion following the application of enhanced bone adhesive remain unknown.

Computed Tomography has been reported to have low sensitivity in the diagnosis of postoperative sternal complications [9]. Measures of sternal separation and micro-motion by ultrasound have demonstrated validity and excellent reliability with intra-class correlation coefficients ranging from 0.90 to 0.93 [10]. In this case report we present the results of a single-center, case reportof sternal separation and micromotion measures obtained by real-time ultrasound that compares conventional wire cerclage alone with adhesive-enhanced sternal closure on a platform of wire cerclage in two patients undergoing median sternotomy for cardiac surgery.

\section{Method}

\section{Patients and study design}

Two patients undergoing first-time cardiac operations through a median sternotomy at a tertiary hospital in Australia who were age and sex matched participated in this study. Inclusion criteria mandated that both adult patients underwent elective operations and were capable of informed consent.

Both patients were treated with standard pain management protocols. Healthcare professionals were blinded to the interventionand were requested to follow routine "sternal precautions" according to the institution. The protocol for this study received ethics approval (ID: HREC 2011.240). Written informed consent was obtained from each participant.

Correspondence to: Dr. Doa El-Ansary, The University of Melbourne, Department of Physiotherapy, Level 7, Alan Gilbert Building, Parkville 3010, Australia, Tel: +6138344 6171; Fax:+6138344 4188; E-mail: d.el-ansary@unimelb.edu.au

Key words: sternum, cardiac surgery, ultrasound, post-operative care

Received: September 12, 2015; Accepted: October 30, 2015; Published: November 03, 2015 


\section{Surgical technique}

Each patient underwent sternal closure using 7-wire interrupted cerclage with 12-gauge stainless steel. Use of bone wax or other hemostatic agents was prohibited. In the adhesive-enhanced closure patient, trabecular bone was cleaned with a sterile brush and irrigated with saline solution. The Kryptonite ${ }^{\mathrm{TM}}$ bone adhesive was mixed for 4 minutes, and then 3 to $6 \mathrm{~mL}$ was applied as a thin layer to each hemisternae at the trabecular interface in compliance with the protocol described previously in a technical report by Fedak and colleagues [2]. Bone and soft-tissue approximation was identical in each patient [2].

\section{Outcome measures}

Sternal separation and micro-motion: This primary outcome was measured by real-time ultrasound. Ultrasound footage was captured with a SonoSite M-Turbo device (SonoSite Australasia Pty Ltd, Australia) using a linear array transducer (15-16 MHz). The transducer was placed directly over the participants' skin or hydrocolloid sternal wound dressing, in the region of the mid sternum corresponding to the fourth intercostal space.

Sternal micro-motion was measured $(\mathrm{mm})$ using the linear calliper function of the software program, such that each end of the calliper was positioned on the respective sternal edges to measure the separation and micro-motion in the coronal (lateral displacement) and the sagittal plane (antero-posterior movement). In this studyoverlap of the sternal edges in the coronal plane (horizontal measure) was recorded as positive and sternal separation was recorded as negative.

Visual Analogue Scale (VAS): The VAS is an established tool for measuring pain. It was selected due to its utility in the cardiac population [11]. This utilizes a $10 \mathrm{~cm}$ horizontal VAS, which had endpoint descriptors of no pain (equivalent to 0 ) and worst imaginable pain (equivalent to 10). Patients were instructed to complete the VAS with each task.

Sternal Instability Scale (SIS): The SIS is a manual physical examination that aims to assess the stability of the sternum. It is based on a 4-point scale that is anchored by agrade of 0 that corresponds to a clinically stable sternum and a grade 3 that corresponds to a completely separated sternum [12]. Previous research has shown that the SIS is both a valid and reliable clinical tool for establishing the stability of the sternum. It has demonstrated excellent inter and intra-rater reliability, with intra-class correlation coefficients of 0.97 and 0.98 respectively [12].
Both patients were asked to perform upper limb movements (unilateral arm elevation, bilateral arm elevation) and a cough three times. Measures were taken with each task repetition and the mean was recorded. Measures were taken by the chief investigator at three time points: day 1, 6 weeks and 3 months post-operatively.

\section{Results}

Both patients were male and aged 60 (patient 1) and 62 (patient 2) years. Patient one had a mitral valve replacement and patient two had an aortic valve replacement. They were comparable with respect to both demographic and surgical domains.

\section{Patient 1 (wire cerclage)}

The micro-motion of the sternal edges as quantified by realtime ultrasound and patient reported pain both reduced overtime. Ultrasound imaging revealed an overlap of the sternal edges in the coronal plane with one edge slighter higher than the other at rest and during all tasks. Table 1 reports the sternal separation and micromotion measures for patient 1 in the coronal and sagittal planes, for each of the tasks. Figure 1 depicts real-time ultrasound images during a cough at the three measurement time points.

Pain reported measures ranged from 4 to $7 ; 3$ to 5 and 1 to 4 out of 10 for the respective three time points (Table 1). Unilateral upper limb elevation and cough were reported to produce the most pain. Physical examination of the sternum revealed a clinically stable sternum (grade 0 ) at each time point.

\section{Patient 2 (adhesive-enhancedsternal closure on a platform of wire cerclage)}

The micro-motion of the sternal edges as quantified by real-time ultrasound and patient reported pain both markedly reduced in the initial post-operative period prior to discharge from the hospital. There was no sternal separation ormicro-motionof the sternal edges post-operatively and the sternum exhibited continuous cortical bone and appeared to be united as observed by real-time ultrasound (Table 1). Figure 2 depicts real-time ultrasound images during a cough at the three measurement time points.

Pain reported measures ranged from 0 to $3 ; 0$ to 1 and 0 out of 10 for the respective three time points (Table 1). Unilateral upper limb elevation and cough was reported to produce the most pain. Patient 2 also reported that the associated pain was localised to the left $4^{\text {th }}$ sternocostal joint. On physical examination the corresponding joint

Table 1. Mean sternal separation and micro-motion(millimeters);mean patient reported pain over a 3-month period.

\begin{tabular}{|c|c|c|c|c|c|c|c|c|c|c|c|c|c|c|c|}
\hline \multirow{2}{*}{\begin{tabular}{|c|} 
Patient 1 \\
Micro-motion
\end{tabular}} & \multicolumn{5}{|c|}{ Day1 post-operatively } & \multicolumn{5}{|c|}{6 weeks post-operatively } & \multicolumn{5}{|c|}{3 months post-operatively } \\
\hline & Rest & DI & UUE & BUE & $\mathrm{C}$ & Rest & DI & UUE & BUE & $\mathrm{C}$ & Rest & DI & UUE & BUE & $\mathrm{C}$ \\
\hline - Coronal & +1.1 & +0.9 & +0.3 & +0.7 & +0.6 & +0.9 & +0.8 & +0.5 & +0.8 & +0.5 & +0.9 & +0.9 & +0.8 & +0.8 & +0.8 \\
\hline - $\quad$ Sagittal & +1.7 & +1.9 & +1.8 & +1.7 & +1.8 & +1.6 & +1.7 & +1.8 & +1.8 & +1.8 & +1.5 & +1.5 & +1.8 & +1.5 & +1.8 \\
\hline Pain (VAS) & 4 & 4 & 6 & 4 & 7 & 3 & 3 & 4 & 3 & 5 & 0 & 1 & 3 & 3 & 4 \\
\hline Patient 2 & \multicolumn{5}{|c|}{ Day 1 post-operatively } & \multicolumn{5}{|c|}{6 weeks post-operatively } & \multicolumn{5}{|c|}{3 months post-operatively } \\
\hline Micro-motion & Rest & DI & UUE & BUE & $\mathrm{C}$ & Rest & DI & UUE & BUE & $\mathrm{C}$ & Rest & DI & UUE & BUE & $\mathrm{C}$ \\
\hline - Coronal & 0 & 0 & 0 & 0 & 0 & 0 & 0 & 0 & 0 & 0 & 0 & 0 & 0 & 0 & 0 \\
\hline - $\quad$ Sagittal & 0 & 0 & 0 & 0 & 0 & 0 & 0 & 0 & 0 & 0 & 0 & 0 & 0 & 0 & 0 \\
\hline Pain (VAS) & 0 & 1 & 3 & 1 & 3 & 0 & 0 & 1 & 0 & 1 & 0 & 0 & 0 & 0 & 0 \\
\hline
\end{tabular}

DI: deep inspiration; UUE: unilateral upper limb elevation; BUE: bilateral upper limb elevation; C: cough; VAS: Visual Analogue Scale 


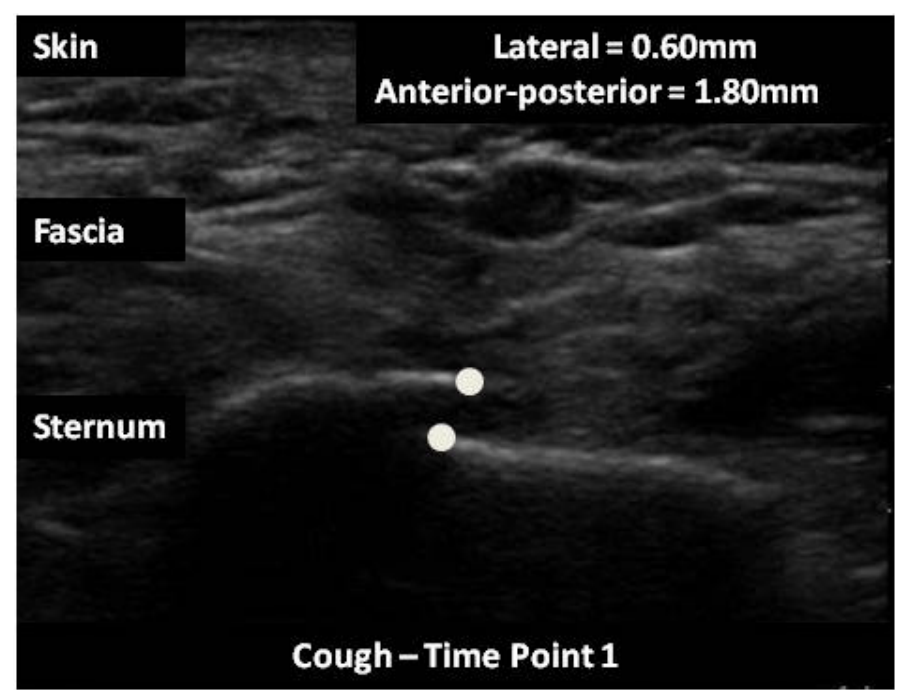

Figure 1. Patient 1(wire cerclage sternal closure) - ultrasound imageduring a cough, day 1 post-operatively. The distance between the sternal edges is $0.60 \mathrm{~mm}$ (lateral) and $1.80 \mathrm{~mm}$ (antero-posterior).

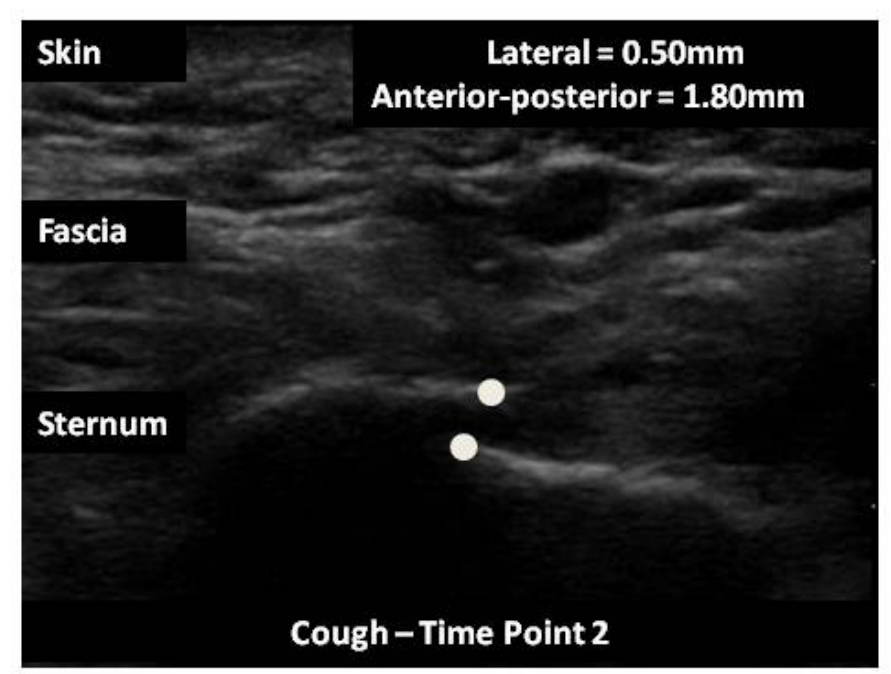

Figure 2. Patient 1 (wire cerclage sternal closure) - ultrasound image during a cough, 6 weeks post-operatively. The distance between the sternal edges is $0.50 \mathrm{~mm}$ (lateral) and $1.80 \mathrm{~mm}$ (antero-posterior)

was tender on palpation. Physical examination of the sternum revealed a clinically stable sternum (grade 0 ) at each time point.

\section{Discussion}

This report measured sternal separation and micro-motion of the hemisternae following a median sternotomy for cardiac surgery in two patients with two varying sternal closure techniques: wire cerclage alone (patient 1) and adhesive-enhanced sternal closure on a platform of wire cerclage (patient 2).

Whilst patient one demonstrated a reduction in reported pain as well as sternal separation and micro-motion over time, at three months post-operatively, the sternum showed no signs of radiological bony union in the coronal and sagittal planes. This is consistent with prior research that reported $50 \%$ of patients following cardiac surgery exhibited signs of complete healing as evidenced by a continuum of cortical bone bridging the entire length of the sternotomy on CT imaging at 6 months post-operatively (9). Alternatively, patient two demonstrated radiological bony union at all three time points, with no recorded sternal separation of micro-motion measured by ultrasound, in both the coronal and sagittal planes. This suggests that adhesive-enhanced sternal closure on a platform of wire cerclage may offer increased sternal stability post-operatively, when compared to conventional wire cerclage sternal closure alone. Additionally, patient two reported less pain at each time point compared to patient one suggesting that there may be a correlation between sternal stability and pain. As such, adhesive-enhanced sternal closure may facilitate patient recovery and return to optimal function.

Sternal separation has also been noted on CT imaging and if small in magnitude has been correlated with a clinically stable sternum [9]. This finding was consistent with our assessment which demonstrated a clinically stable sternum (SIS). A diagnosis of sternal instability may require a review of the clinical presentation that includes patient reported symptoms of pain/discomfort and instability; physical examination as well as ultrasound imaging. This observational study

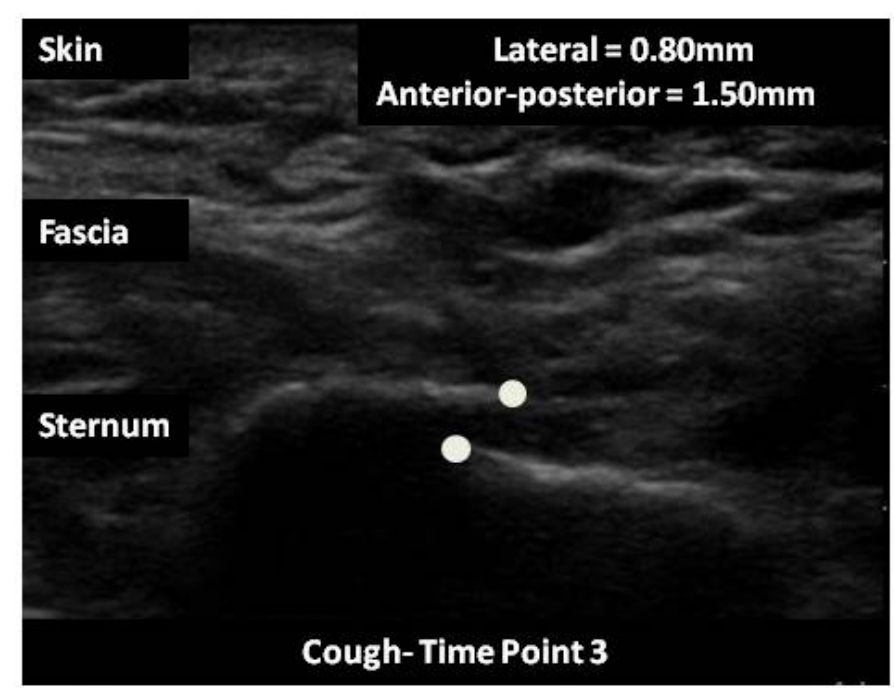

Figure 3. Patient 1(wire cerclage sternal closure) - ultrasound image during a cough, 3 months post-operatively. The distance between the sternal edges is $0.80 \mathrm{~mm}$ (lateral) and $1.50 \mathrm{~mm}$ (antero-posterior)

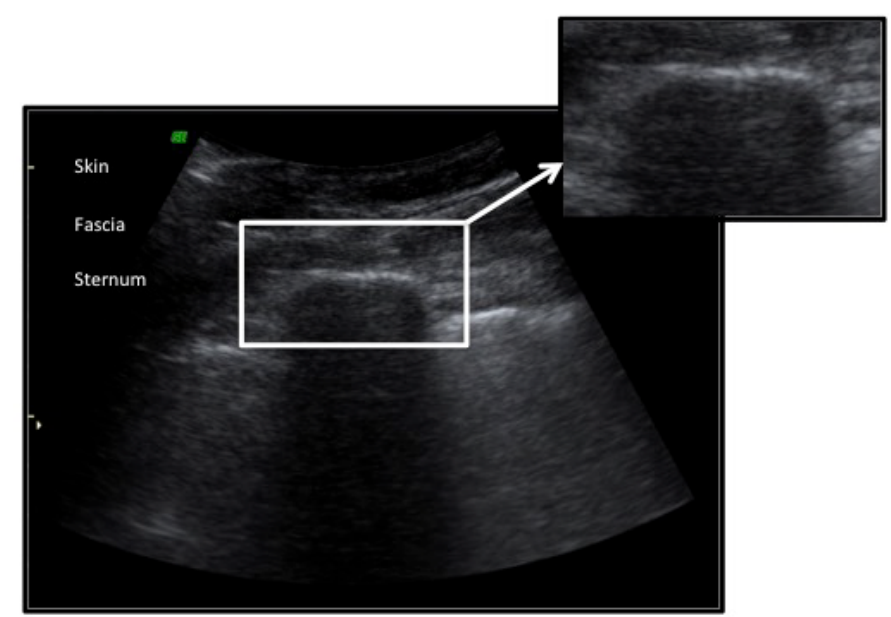

Figure 4. Patient 2(enhanced-adhesive on a platform of wire cerclage sternal closure) during a cough at day 1 post-operatively. No sternal separation or micro-motion of the sternal edges. Bone union evident. 


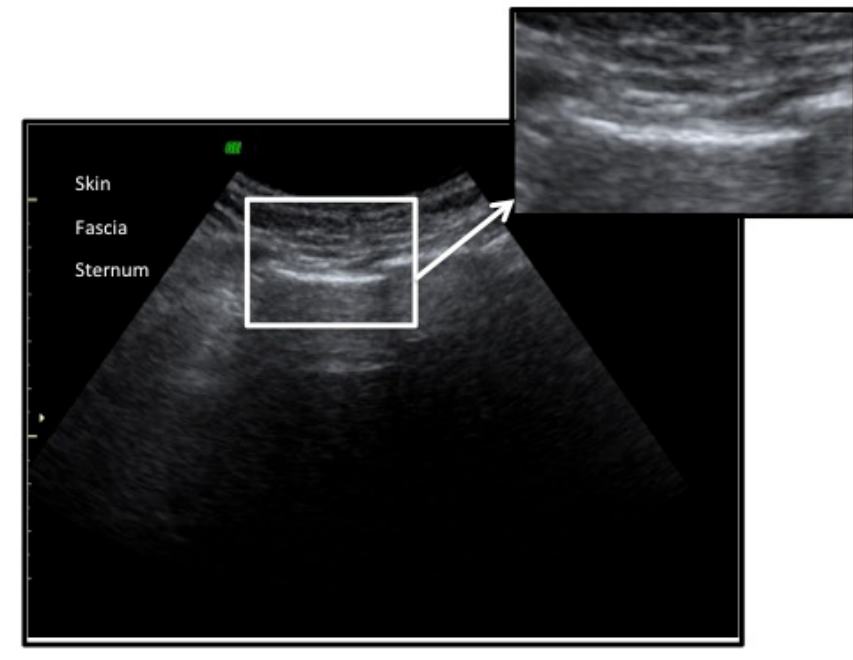

Figure 5. Patient 2(enhanced-adhesive on a platform of wire cerclage sternal closure) during a cough at 6 weeks post-operatively. Bone union evident.

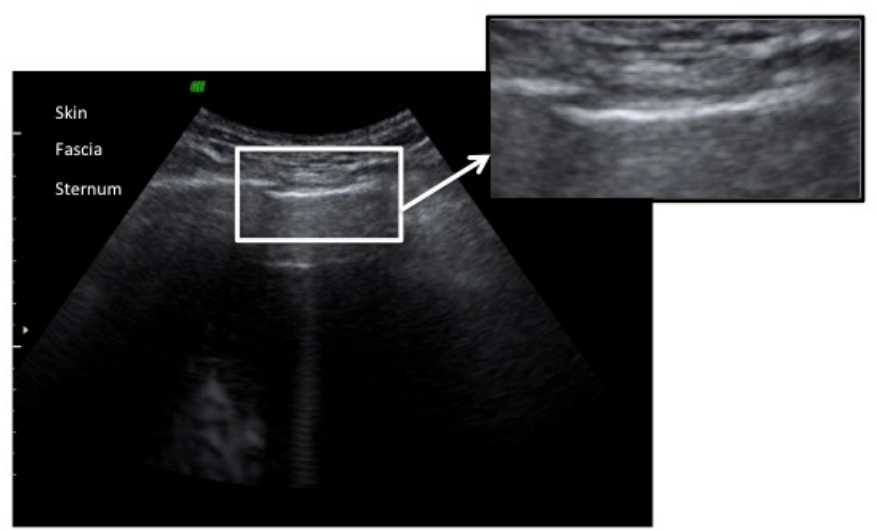

Figure 6. Patient 2(enhanced-adhesive on a platform of wire cerclage sternal closure) during a cough at 3 months post-operatively. Bone union evident.

also confirmed prior research findings of multi-planar micro-motion of the sternal edges with motion in the coronal and sagittal planes depicted on real-time ultrasound [8]. Whilst micro-motion in the longitudinal direction may be present this cannot be measured with 2D ultrasound imaging [8].

Real-time ultrasound is a non-invasive, valid and reliable measurement tool for quantification of sternal separation and micro-motion; and evaluation of sternal closure techniques. It can be performed within the acute setting immediately post-operatively. Future research investigating sternal closure techniques by ultrasound in particular adhesive-enhanced closure is warranted in a larger population.

\section{Consent}

Written informed consent was obtained from the patient for publication of this case report and any accompanying images at the time of the study.

\section{References}

1. Fedak PW, Kieser TM, Maitland AM, Holland M, Kasatkin A, et al. (2011) AdhesiveEnhanced Sternal Closure to Improve Postoperative Functional Recovery: A Pilot, Randomized Controlled Trial. Ann Thorac Surg 92: 1444 -1450. [Crossref]

2. Fedak PW, Kasatkin A (2011) Enhancing sternal closure using Kryptonite bone adhesive: technical report. Surg Innov 18: NP8-11. [Crossref]

3. Fedak PW1, Kolb E, Borsato G, Frohlich DE, Kasatkin A, et al. (2010) Kryptonite bone cement prevents pathologic sternal displacement. Ann Thorac Surg 90: 979-985. [Crossref]

4. Mcgregor WE, Trumble DR, Magovern JA (1999) Mechanical analysis of midline sternotomy wound closure. J Thorac Cardiovasc Surg 117: 1144-1150. [Crossref]

5. Tarzia V, Carrozinni M, Bortolussi G, Buratto E, Beiko J, et al. (2014) Impact of vacuum-assisted closure therapy on outcomes of sternal wound dehiscence. Interact Cardiovasc Thorac Surg 19: 70-75. [Crossref]

6. Arnoni RT, Danta DC, Nigro NC, Abdulmassih NC (2013) Assessment of sternal closure using titanium plate. Rev Bras Cir Cardiovasc 28: 386-390. [Crossref]

7. Pericleous A, Dimitrakakis G, Photiades R, Von Oppell UO (2015) Assessment of vacuum-assisted closure therapy on the wound healing process in cardiac surgery. Int Wound J. [Crossref]

8. El-Ansary D, Waddington G, Adams R (2009) Sternal instability during arm elevation observed as dynamic, multiplanar separation. International Journal of Therapy and Rehabilitation 16: 609-614.

9. Bitkover CY, Cederlund K, Aberg B, Vaage J (1999) Computed tomography of the sternum and mediastinum after median sternotomy. Ann Thorac Surg 68: 858-863. [Crossref]

10. El-Ansary D1, Waddington G, Adams R (2007) Measurement of non-physiological movement in sternal instability by ultrasound. Ann Thorac Surg 83: 1513-1516. [Crossref]

11. Lapier TL, Wilson B (2007) Prevalence and severity of symptoms in patients recovering from coronary artery bypass surgery. Acute Care Perspectives 16: 10-15.

12. EL-Ansary D, Waddington G, Adams R (2007) Trunk stabilization exercises reduce sternal separation in chronic sternal instability after cardiac surgery: a randomized cross-over trial. Aust J Physiother 53: 255-260. [Crossref]

Copyright: $@ 2015$ El-Ansary D. This is an open-access article distributed under the terms of the Creative Commons Attribution License, which permits unrestricted use, distribution, and reproduction in any medium, provided the original author and source are credited. 